\title{
Developments in the control of growing conditions in Dutch glasshouse horticulture
}

\author{
E. Kooistra
}

Glasshouse Crops Research and Experiment Station, P.O. Box 8, 2670 AA Naaldwijk, Netherlands

\section{Introduction}

Glasshouse horticulture is of great economic significance in the Netherlands. In 1985 the acreage amounted to 9000 ha. Important also was the increase of production per unit of area. In the period from 1975 to 1985 it increased with $4 \%$ per annum for the most important crops. Improved growing conditions were highly responsible for this increase.

Glasshouse cultivation is characterized by the possibility to influence the growing conditions of crops more or less independently from the outside climate. This can be achieved by e.g. heating, watering and ventilating. In this respect it is essential to know the requirements of the different crops. Only this knowledge makes it possible to pursue growing conditions which are the most desirable in terms of growth, production and quality and which are at the same time economically justified. With respect to insight into the demands of crops a number of drastic developments have taken place in recent years. However, improvements in the technology of glasshouse climate control were even more spectacular.

In the present paper some remarks will be given regarding the progress made with respect to the control of growing conditions in the glasshouse. Furthermore, attention will be paid to some important research items and perspectives.

\section{Growing factors}

The most important factors for the growth of a crop are light, $\mathrm{CO}_{2}$, air temperature and humidity, soil temperature, water and nutrients. The factor light is the most difficult to influence, at least in an economic way. In most cases the application of artificial light is not economically justified.

Only a small part (about $5 \%$ ) of the natural radiation is utilized for photosynthesis of the plants. Roughly one third is used for heating up the glasshouse interior, and slightly less than two thirds of the incoming energy is used for transpiration of the crop. Especially in the winter months the available amount of light constitutes the minimum factor. Therefore, in commercial glasshouse horticulture much attention is being paid to the amount of light entering the glasshouse.

The other factors mentioned can be influenced in the glasshouse in a more simple way although the conditions in the root zone are less regulable than the conditions in the aerial environment. However, also complete control of the aerial climate is 
fictitious because it is strongly dependent on the outside climate. Nevertheless, considerable progress has been made in this field during recent years.

\section{Control of growing factors}

\section{Heating and ventilating}

Heating by a heating installation and ventilating by means of ventilators are possibilities to affect the glasshouse climate. These measures have been available to growers a long time. Initially, both were used for the control of air temperature. Ventilation, however, not only involves removal of heat but also the output of water vapour. Circumstances may occur in which the opening of the ventilators is utilized to stimulate transpiration. Already in the 1950 s tomatoes were grown in heated glasshouses with open windows when the weather was mild and dull, thus controlling and stimulating crop growth. Also other methods were applied to stimulate transpiration in light-deficient periods, for instance by application of a 'heat blast'. The manual control of temperature and ventilation remained defective because of continuous fluctuations in the outside conditions.

In the course of the 1960 s the above situation changed with the advent of automatic analogue control equipment which found general acceptance in commercial practice. Besides saving of labour, the analogue control equipment had two advantages: undesirable fluctuations in the glasshouse climate could be avoided more easily and new insights into crop requirements could be applied more easily. Experiments were carried out, for example, with the 'delta- $X$ ' or moisture deficit control in which ventilation took place depending on air humidity (Heijna, 1975). Various control systems were realized: initially the proportional controls to maintain a glasshouse temperature without too many fluctuations and, subsequently, proportional-integrating controls in order to minimize adjusted and realized values. These systems included facilities to create light-intensity-dependent day temperatures and day-night transitions by means of a time clock.

The next stage began when the analogue control was replaced by the process computer. In 1974 a process computer was installed at our Research Station in a glasshouse consisting of 24 compartments and designed especially for glasshouse climate research (van de Vooren, 1975; van de Vooren \& Koppe, 1975). Soon afterwards, in 1975, the first computer was introduced into commercial practice. Nowadays the number of computers for the control of the glasshouse climate is more than 4000 .

The advantages mentioned above when manual control was replaced by analogue control were even more apparent at the switch to digital control. The various factors could be controlled more effectively by means of these process computers; moreover, it became more simple to aim at the optimum combination of factors. New insights and more advanced control algorithms could be made operative rapidly by adapting the software (Udink ten Cate \& van de Vooren, 1981). This development gained an extra dimension by the attempts to improve the utilization of energy which were initiated after the outbreak of the energy crisis in 1973. Since then new control strategies have been developed and tested. 


\section{Energy saving}

Energy saving had a profound impact on climate control strategies. Energy conservation was achieved by heating more economically. This involved the maintenance of adapted temperature regimes, reduction of ventilation whenever possible, application of thermal screens, etc. These measures yielded good results. Besides the direct saving of energy, the increase in production continued unabated. The overall result was that since the outbreak of the energy crisis the energy consumption per unit of product has been reduced by about $50 \%$. However, new problems arose which initiated further research.

As a result of the application of thermal screens, greater temperature differences occurred in the glasshouse, both in horizontal and in vertical direction. Adaptation in the heating system appeared necessary. Application of thermal screens was also accompanied by loss of light, which had to be restricted as much as possible. Opening and closing of the screens needed special attention to avoid too great climate transitions. Control systems were developed for the opening and closing of the screens in which the process computer was engaged.

Reduced ventilation led to renewed attention for the application of additional $\mathrm{CO}_{2}$ (Heij \& van Uffelen, 1984), initiated after the observation that little ventilation may result in an atmospheric $\mathrm{CO}_{2}$ concentration below the desired value, even if additional $\mathrm{CO}_{2}$ is applied (Heij \& Schapendonk, 1984). Moreover, as a result of reduced ventilation the risk of high concentrations of harmful gases in the glasshouse increased. Special attention is being paid to gases as $\mathrm{NO}_{\mathrm{x}}$ in cases where the $\mathrm{CO}_{2}$ is applied as off-gas from the heating boiler (Wolting et al., 1985). Reduced ventilation may also have a drastic effect on moisture transport from the glasshouse and on crop transpiration (Bakker, 1984a, 1984b, 1986; de Graaf, 1985).

Higher air humidities and reduced transpiration rates may have serious consequences for ion uptake and growth of the crops, and for yield and quality of the harvested products. The occurrence of diverse physiological disorders increases as the conditions for a proper transpiration of the crop become less favourable (Bakker, 1984c). Consequently, study of the relationship between nutrition of the plant and growth and product yield and quality under low-energy conditions, has received high priority.

\section{Growing on artificial substrates}

In view of the developments described above, it is a fortunate coincidence that cultivation on artificial substrates has been developed. This growing method enables, to a greater extent than was possible before, control of conditions in the root environment. Growing on artificial substrates almost exclusively takes place on rockwool. This development started in 1975 and has since then strongly increased in the Netherlands. At this moment about 2000 ha are used for plant cultivation on artificial substrates. This concerns mainly the crops tomato, cucumber, sweet pepper, and eggplant. Until now the floriculture has remained behind, but also in this branch interest is increasing.

One of the most important aspects of this growing method is the regular application of a nutrient solution. Basic data and recipes for the composition of nutrient so- 


\section{E. KOOISTRA}

lutions applied to various crops and for their composition in the root vicinity have been developed in our Research Station. For the control of the application of water and nutrients a process computer has been developed. Several aspects of growing vegetables in substrates have been described by Sonneveld \& Welles (1984). Significant production enhancements, varying from 10 to $15 \%$, have already been realized. Because also quality is strongly related to the nutritional status, quality improvement can be reached by using the rockwool growing system. These aspects need further study in the near future. For this purpose an extensive research programme has been set up.

\section{Automated management information systems}

In recent years it was recognized that control of the growing conditions is strongly determined by the judgement of the grower and his ability to utilize the resources available. This means that all factors which are important for the ultimate farm outcome should be controlled in their mutual coherence. Consequently, the need for adequate management information systems is increasingly felt (Ammerlaan, 1984). A start has been made in recording and processing of data such as yields, energy consumption, labour management (Ammerlaan et al., unpublished). Connected with, for example, the (external) auction computer and the (internal) climate computer, the business computer will be able to play a central role. It is also possible to improve the already existing forms of farm comparison, provided that input of data is standardized. When growers have their data processed on a central computer, comparative results can be received very quickly, enabling each participating grower a better understanding of the strong and weak aspects of his nursery.

\section{Future research and developments}

In the near future much knowledge will have to be gathered in order to optimize the control of the aerial climate. Central issues will be the fundamental knowledge of crop physiology and application of this knowledge to improve growth and development, and thorough study of the physical behaviour of a glasshouse with a crop and the inter-relations between the various growing factors. Besides possibilities offered by the different soilless culture methods, optimum conditions in the root environment have to be assessed. Efforts will have to be directed towards continuous regulation in order to achieve both quantitatively and qualitatively optimum supply of water and nutrients. In connection with this, adequate measuring methods will have to be developed.

Optimization requires a detailed insight into the relationships between growing factors and additional returns; also additional costs should be taken into account. An important evolution is the development of models, for example for the regulation of $\mathrm{CO}_{2}$ application (Nederhoff \& Schapendonk, 1985). A treatment according to models requires subsequent development of proper programmes on the basis of which the desired regulation and growth control can be realized. Process control should thus be further perfected. 
Based on the above, our conclusion may be that we can expect:

- continuous improvement of the process control regarding glasshouse climate and root environment, probably by a connection between computer regulation of the aerial climate and that of the root environment;

- optimum utilization of the production strategy by connecting the process control to internal and external information via the business computer.

However, improvements which must be regarded as potentially very substantial, will probably materialize only gradually. It is therefore reasonable to presume that in the coming decade the rate of production increase and quality improvement will maintain at least the same level as has been realized in the previous decade.

\section{References}

Ammerlaan, J. C. J., 1984. Development in management information systems in the glasshouse horticulture in the Netherlands. Acta Horticulturae 155: 235-242.

Bakker, J. C., 1984a. Water vapour transport from a greenhouse by ventilation. I. Effects on climate. Acta Horticulturae 148: 535-542.

Bakker, J. C., 1984b. Water vapour transport from a greenhouse by ventilation. II. Effects on cucumber. Acta Horticulturae 148: 543-546.

Bakker, J. C., 1984c. Physiological disorders in cucumber under high humidity conditions and low ventilation rates in greenhouses. Acta Horticulturae 156: 257-264.

Bakker, J. C., 1986. Measurements of canopy transpiration or evapotranspiration by means of a simple vapour balance model. Agricultural and Forest Meteorology 37: 133-141.

Graaf, R. de, 1985. The influence of thermal screening and moisture gap on the transpiration of glasshouse tomatoes during the night. Acta Horticulturae 174: 57-66.

Heij, G. \& A. H. C. M. Schapendonk, 1984. $\mathrm{CO}_{2}$ depletion in greenhouses. Acta Horticulturae 148: 351 355 .

Heij, G. \&. J. A. M. van Uffelen, 1984. Effect of $\mathrm{CO}_{2}$ concentration on growth and production of glasshouse vegetable crops. Acta Horticulturae 148: 591-595.

Heijna, B. J., 1975. Delta X - a new control system. Acta Horticulturae 46: 13-19.

Nederhoff, E.M. \& A. H. C. M. Schapendonk, 1985. Effects of environment conditions on growth and production of cucumber; comparison between empirical and simulation data. Acta Horticulturae 174: 251-258.

Sonneveld, C.\& G. W. H. Welles, 1984. Growing vegetables in substrates in the Netherlands. Proceedings of the 6th Congress of the International Society for Soilless culture (Lunteren), p. 613-632.

Udink ten Cate, A. J. \& J. van de Vooren, 1981. Adaptive systems in greenhouse climate control. Proceedings of the 8 th IFAC World Congress (Kyoto), p. 9-15.

Vooren, J. van de, 1975. A computer for crop research and climate control in glasshouses. Acta Horticulturae 51: 169-174.

Vooren, J. van de \& R. Koppe, 1975. The climate glasshouse at Naaldwijk. Netherlands Journal of Agricultural Science 23: 238-247.

Wolting, H. G., E. A. M. van Remortel \& N. van Berkel, 1985. Air quality in greenhouses with and without $\mathrm{CO}_{2}$ enrichment. Acta Horticulturae 184: 351-357. 